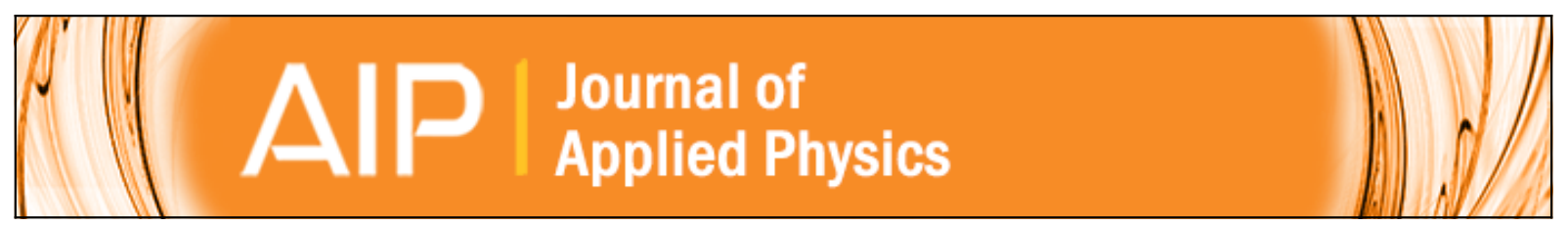

\title{
Magnetoelectric nonlinearity in magnetoelectric laminate sensors
}

Liangguo Shen, Menghui Li, Junqi Gao, Ying Shen, J. F. Li, D. Viehland, X. Zhuang, M. Lam Chok Sing, C.

Cordier, S. Saez, and C. Dolabdjian

Citation: Journal of Applied Physics 110, 114510 (2011); doi: 10.1063/1.3665130

View online: http://dx.doi.org/10.1063/1.3665130

View Table of Contents: http://scitation.aip.org/content/aip/journal/jap/110/11?ver=pdfcov

Published by the AIP Publishing

\section{Articles you may be interested in}

Flux distraction effect on magnetoelectric laminate sensors and gradiometer

J. Appl. Phys. 114, 134104 (2013); 10.1063/1.4824184

High-resolution current sensor utilizing nanocrystalline alloy and magnetoelectric laminate composite Rev. Sci. Instrum. 83, 115001 (2012); 10.1063/1.4763570

Comparison of noise floor and sensitivity for different magnetoelectric laminates

J. Appl. Phys. 108, 084509 (2010); 10.1063/1.3486483

Ring-type electric current sensor based on ring-shaped magnetoelectric laminate of epoxy-bonded Tb 0.3 Dy 0.7 Fe 1.92 short-fiber/NdFeB magnet magnetostrictive composite and $\mathrm{Pb}(\mathrm{Zr}$, Ti)O 3 piezoelectric ceramic J. Appl. Phys. 107, 09D918 (2010); 10.1063/1.3360349

Circumferential-mode, quasi-ring-type, magnetoelectric laminate composite—a highly sensitive electric current andor vortex magnetic field sensor

Appl. Phys. Lett. 86, 182506 (2005); 10.1063/1.1923184

MIT LINCOLN

LABORATORY CAREERS

Discover the satisfaction of innovation and service

to the nation
- Space Control

- Air \& Missile Defense

- Communications Systems \& Cyber Security

- Intelligence, Surveillance and

Reconnaissance Systems

- Advanced
Electronics
- Tactical Systems
- Homeland
Protection
- Air Traffic Control

LINCOLN LABORATORY

MASSACHUSETTS Institute OF TeChNOLOGY

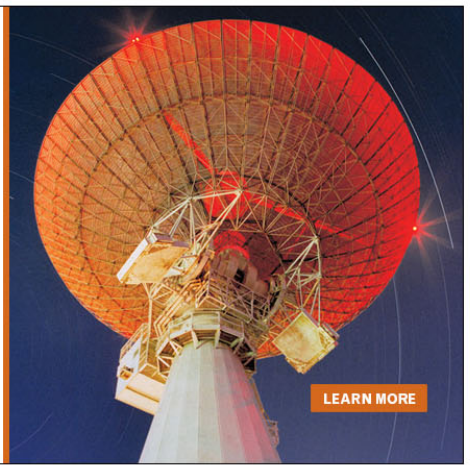




\title{
Magnetoelectric nonlinearity in magnetoelectric laminate sensors
}

\author{
Liangguo Shen, ${ }^{1, a)}$ Menghui Li, ${ }^{1}$ Junqi Gao, ${ }^{1}$ Ying Shen, ${ }^{1}$ J. F. Li, ${ }^{1}$ D. Viehland, ${ }^{1}$ X. Zhuang, ${ }^{2}$ \\ M. Lam Chok Sing, ${ }^{2}$ C. Cordier, ${ }^{2}$ S. Saez, ${ }^{2}$ and C. Dolabdjian ${ }^{2}$ \\ ${ }^{1}$ Department of Materials Science and Engineering, Virginia Tech, Blacksburg, Virginia 24061, USA \\ ${ }^{2}$ Groupe de Recherche en Informatique, Image, Automatique et Instrumentation de Caen (GREYC), CNRS \\ UMR 6072-ENSICAEN and University of Caen, Caen Cedex, 14050, France
}

(Received 10 February 2011; accepted 27 October 2011; published online 5 December 2011)

\begin{abstract}
A nonlinearity in the magnetoelectric coefficient, $\alpha_{M E}^{\text {Nonlin }}$, of Metglas $/ \mathrm{Pb}(\mathrm{Zr}, \mathrm{Ti}) \mathrm{O}_{3}(\mathrm{PZT})$ and Metglas $/ \mathrm{Pb}\left(\mathrm{Mg}_{1 / 3}, \mathrm{Nb}_{2 / 3}\right) \mathrm{O}_{3}-\mathrm{PbTiO}_{3}$ (PMN-PT) laminate sensors has been observed. This nonlinearity was found to be dependent on the dc magnetic bias $\left(H_{\mathrm{dc}}\right)$ and frequency of the ac drive field $\left(H_{\mathrm{ac}}\right)$. The maximum value of $\alpha_{M E}^{\text {Nonlin }}$ for both types of composites was found near the electromechanical resonance. For Metglas/PZT laminates, the maximum occurred under a finite bias of $H_{\mathrm{dc}} \approx 5$ Oe; whereas, for Metglas/PMN-PT, the maximum was found near zero dc bias. One application for $\alpha_{M E}^{\text {Nonlin }}$ is a cross-modulation scheme that can shift low frequency signals to higher frequency to achieve lower noise floor. For Metglas/PMN-PT, $\alpha_{M E}^{\text {Nonlin }}$ has another application: removal of the necessity of a dc bias, which helps to design high-sensitivity sensor arrays and gradiometers. (C) 2011 American Institute of Physics. [doi:10.1063/1.3665130]
\end{abstract}

\section{INTRODUCTION}

Recently, there has been much interest in low-frequency $(\approx 1 \mathrm{~Hz})$, low-power, low-cost, room-temperature magnetic sensors with high sensitivity and low equivalent magnetic noise floors. ${ }^{1,2}$ There are various types of commercial magnetic sensors that have some of these characteristics, but not all. With regards to high sensitivity and low noise floors, superconducting quantum interference devices (SQUIDs) are the most well-known: but, they need to be operated at cryogenic temperature, which makes the system costly and large. ${ }^{3}$ Other smaller and less costly magnetic sensors, such as fluxgates, do not have the required sensitivities and detectable field ranges. ${ }^{4}$ They operate at room temperature, but are not truly passive.

To date, magnetic sensors based on the magneto-electric (ME) effect have been widely investigated. ${ }^{4-10}$ The ME effect is a polarization response to an applied magnetic field, $H$; or conversely, a magnetization response to an applied electric field, $E{ }^{5}$ Researchers have found that two-phase laminate composites which contain magnetostrictive and piezoelectric phase layers laminated together have giant $\mathrm{ME}$ effects. ${ }^{6}$ Generally, the ME response is described by a linear ME voltage coefficient $\alpha_{M E}$, defined as ${ }^{5-7}$

$$
\alpha_{M E}=E / H_{i n c}[\mathrm{~V} / \mathrm{cm}-\mathrm{Oe}]
$$

where $H_{\text {inc }}$ is the incident field to be detected. Values of the linear $\mathrm{ME}$ voltage coefficient of $\alpha_{M E}=20 \mathrm{~V} / \mathrm{cm}-\mathrm{Oe}$ and $\alpha_{M E}=500 \mathrm{~V} / \mathrm{cm}-$ Oe have been reported at low and electromechanical resonance (EMR) frequencies, ${ }^{7}$ respectively, for the trilayer laminate of Metglas $/ \mathrm{Pb}(\mathrm{Zr}, \mathrm{Ti}) \mathrm{O}_{3}(\mathrm{PZT}) /$ Metglas.

Prior investigations have focused on the linear $\mathrm{ME}$ response $^{4-10}$ and constitutive equations. ${ }^{5}$ However, the individual layers are known to have significant piezoelectric ${ }^{8}$

\footnotetext{
a) Author to whom correspondence should be addressed. Electronic mail: shenliangguo@gmail.com.
}

and effective piezomagnetic ${ }^{9}$ nonlinearities. Such nonlinearities are known to be extrinsic, and dependent on external variables, such as frequency and dc electric/magnetic fields. These nonlinearities, in the piezoelectric coefficient, have contributions from domain wall dynamics. ${ }^{10}$ Different regimes of domain wall dynamics are known under different drive ranges: these include both reversible and irreversible domain wall displacements. In ME laminate systems with significant magnetoelectric coupling, inherent non-linearities induced in one phase should be transferred to the other. An equivalent model for the ME laminate can be reached based on constitutive piezoelectric, piezomagnetic, and motion equations. ${ }^{5,11}$ The ME voltage $(d V / d H)$ and ME charge $(d Q / d H)$ coefficients in the L-T mode can be deduced from the 1-D Mason's equivalent model as

$$
\begin{aligned}
\frac{d V}{d H} \approx & \left(\frac{n(1-n) d_{33, m} g_{31, p}}{n s_{11, p}^{E}\left(1-k_{31, p}^{2}\right)+(1-n) s_{33, m}^{H}}\right) t_{\text {lam }} \cdot[\mathrm{V} / \mathrm{Oe}] \\
\frac{d Q}{d H} \approx & \left(\frac{d_{33, m} h_{31, p} \varepsilon_{33}^{S} s_{33, m} s_{11, p}}{\left(n s_{11, p}+(1-n) s_{33, m}\right) t_{\text {lam }}+s_{33, m} s_{11, p} h_{31}^{2} \varepsilon_{33}^{S} l w}\right) \\
& \times l w t_{\text {lam }} \cdot[\mathrm{C} / \mathrm{Oe}]
\end{aligned}
$$

where $s_{11, p}^{E}$ and $s_{33, m}^{H}$ are the elastic compliances of the piezoelectric and magnetostrictive layers, $d_{33, m}$ the effective linear longitudinal piezomagnetic coefficient, $g_{31, p}$ the transverse piezoelectric voltage coefficient, $k_{31, p}$ the piezoelectric coupling coefficient, $n$ the thickness ratio of the piezoelectric and magnetostrictive layers, $l$ and $w$ are length and width of the laminate, and $t_{\text {lam }}$ is the total thickness of the laminate. ${ }^{12}$ Clearly, these formulas can be extended to nonlinear regimes, given that the piezomagnetic and piezoelectric coefficients involved are allowed to be nonlinear.

The best ME laminate composites, as magnetic sensors, achieve an intrinsic equivalent magnetic noise floor of 20 
$\mathrm{pT} / \sqrt{ } \mathrm{Hz}$ at $1 \mathrm{~Hz}$ in a good laboratory magnetic environment and at room temperature. ${ }^{13}$ Fluctuations in low frequency noise (i.e., $1 / \mathrm{f}^{\mathrm{n}}$, with $\mathrm{n}$ as an integer) make further decreases in the noise floor increasingly challenging. Previously, ME sensors have used a charge amplifier (CA) detection method to amplify low frequency magnetic signals. ${ }^{14}$ Figure 1 shows a typical noise model for such a ME sensor detection unit, where $v_{\mathrm{n}}$ and $i_{\mathrm{n}}$ are the equivalent input voltage noise and current noise of the charge amplifier, ${ }^{15}$ respectively; $R_{\mathrm{f}}$ is the noise generated by the feedback resistor of the charge amplifier; while $R_{\text {in }}$ and $\tan (\delta)$ are noises generated by the leakage resistance and dielectric loss factor of the piezoelectric/ dielectric layer, respectively. In this graph, it can be seen that the noise floor at low frequency (such as $1 \mathrm{~Hz}$ ) is around 10 times higher than that at higher frequency (such as 1 $\mathrm{kHz})$.

Three approaches might be taken to mitigate the low frequency magnetic noise floor. The first is to reduce the low frequency noise of the ME sensor detection unit. In this case, the lowest achievable noise floors are limited by physical parameters $\left(R_{\text {in }}\right.$ and $\left.\tan (\delta)\right)$ (Refs. 12 and 15) and the requirement of room-temperature operation. In addition, when operating the sensor in a laboratory environment, external low frequency noises (such as vibration and thermal noises) will also be limiting factors. ${ }^{15-17}$ The second approach is to implement a modulation scheme. ${ }^{18}$ Such a scheme can only mitigate low frequency noise that exists in the charge amplifier, and thus, the internal noise of the sensor and external low frequency noises will still be limitations.

The third approach is to use a cross-modulation scheme. ${ }^{12}$ An implementation of a ME sensor detection unit using such cross modulation can be found in Ref. 12. The low frequency magnetic signal that needs to be detected is an incident field $H_{\text {inc }}$ with a frequency of $f_{1}$, while a drive field $H_{\text {ac }}$ with a frequency of $f_{0}$ is applied to the ME sensor using a drive coil. Cross modulation scheme makes use of the nonlinear ME coefficient $\alpha_{M E}^{\text {Nonlin }}$ of ME sensors, which is defined as

$$
\alpha_{M E}^{\text {Nonlin }}=E /\left(H_{\text {inc }} \times H_{a c}\right) \cdot\left[\mathrm{V} / \mathrm{cm}-\mathrm{Oe}^{2}\right],
$$

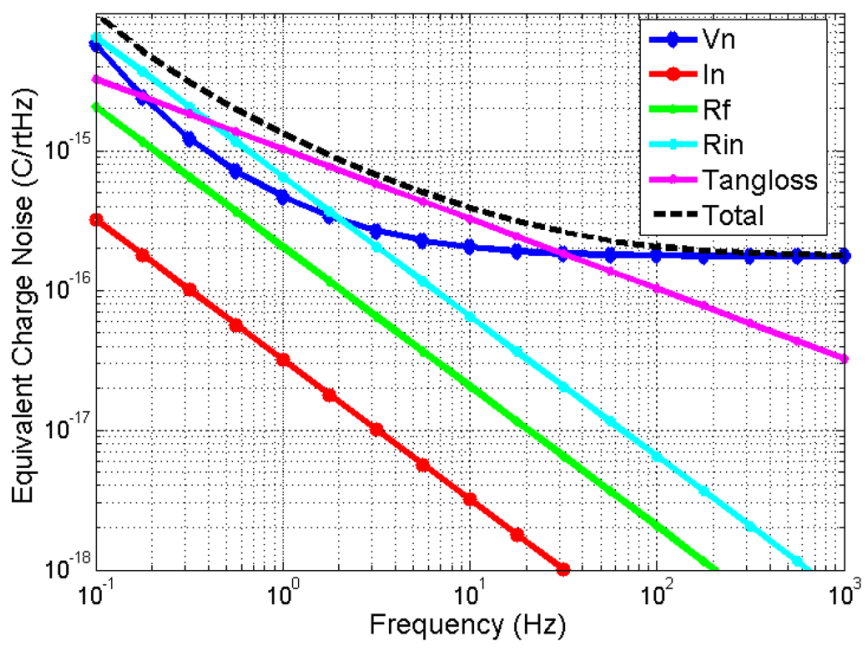

FIG. 1. (Color online) Noise model of charge amplifier based ME sensor detection unit. where $H_{\mathrm{inc}}$ and $H_{\mathrm{ac}}$ are the incident and drive fields, respectively. For a linear ME response, there would be no mixing of these two fields. However, if the ME response is nonlinear, then the output signal of the ME sensor will contain cross modulated fields with frequencies of $\left(f_{0} \pm f_{1}\right)$. Since $f_{0}$ is usually much higher than $f_{1}$, the nonlinear ME coefficient $\alpha_{M E}^{N o n l i n}$ offers the potential to modulate low frequency magnetic signals (at frequency $\left.f_{1}\right)$ to higher frequencies $\left(f_{0} \pm f_{1}\right)$, where the noise floor is much lower, as shown in Fig. 1. The potential exists for intrinsic/environmental noise rejection capabilities that exceed those of the charge amplifier detection method, but this potential is not yet realized. Please note that realization of cross-modulation depends on the magnitude of the nonlinear ME coefficients $\alpha_{M E}^{\text {Nonlin }}$, which as noted above have yet to be determined and understood.

In this paper, the nonlinear ME coefficient $\alpha_{M E}^{\text {Nonlin }}$ of Metglas/PZT and Metglas/ $\mathrm{Pb}\left(\mathrm{Mg}_{1 / 3}, \mathrm{Nb}_{2 / 3}\right) \mathrm{O}_{3}-\mathrm{PbTiO}_{3}$ (PMN-PT) ME laminates is reported for the first time. We show significant values of $\alpha_{M E}^{\text {Nonlin }}$ near the electromechanical resonance frequency for both types of laminates. The maximum value of $\alpha_{M E}^{\text {Nonlin }}$ is achieved near zero dc magnetic bias for Metglas/PMN-PT laminates, but under finite biases for Metglas/PZT.

\section{EXPERIMENTAL APPROACH}

Trilayer ME laminate composites consisting of Metglas and PZT layers, and Metglas and PMN-PT, were fabricated following the procedures previously published. ${ }^{19}$ The Metglas foils were obtained from Vacuumschmelze (Hanau, Germany) and were $27 \mu \mathrm{m}$ in thickness. The foils were cut into a dimension of $8 \mathrm{~cm}$ in length and $1 \mathrm{~cm}$ in width. The PZT fibers were obtained from Smart Material Corp (Sarasota, FL, USA) and were $170 \mu \mathrm{m}$ in thickness. Textured PMN-PT fibers were obtained from Ceracomp (Cheonan, Korea) and were of the same thickness. Both types of piezoelectric fibers were cut to a length of $4 \mathrm{~cm}$ and a width of $0.2 \mathrm{~cm}$.

In the fabrication of the laminates, we first epoxied three layer stacks of Metglas foils together, following prior reports that resulted in enhanced values of $\alpha_{M E}{ }^{18}$ We then deposited Inter-Digitade (ID) electrodes on both sides of the piezoelectric layer which consisted of 5 fibers. Kapton insulating layers were then epoxied on top of both sides. Finally, we assembled ME laminates by bonding 3-layer Metglas stacks on each side of the piezoelectric fiber.

The magnetostriction was characterized by a strain gauge method, where the strain induced by an applied dc magnetic bias was measured by a strain gauge bridge module BCM-1 (Omega: Stamford, CT, USA). The effective piezomagnetic coefficient was then simply calculated from the derivative of the magnetostriction. We next measured the linear magnetoelectric coefficient $\alpha_{M E}$ using a method previously published. ${ }^{20}$ In this method, the voltage induced across the piezoelectric by application of an ac magnetic field was measured at various frequencies and dc magnetic biases.

Finally, we measured the nonlinear ME coefficient $\alpha_{M E}^{\text {Nonlin }}$. We applied both an incident field at a frequency $f_{1}$ using a Helmholtz coil and a drive field at a frequency $f_{0}$ using a drive coil, and then measured the cross-modulation 
fields having frequencies of $\left(f_{0} \pm f_{1}\right)$ using an SR-785 dynamic signal analyzer (Stanford Research Systems: Sunnyvale, CA). We converted the cross-modulation fields to $\alpha_{M E}^{\text {Nonlin }}$ (with unit of $\mathrm{V} / \mathrm{cm}-\mathrm{Oe}^{2}$ ): which was done by knowing the applied incident and drive fields, and the spacing of the ID electrodes. The cross-modulation fields $\left(f_{0} \pm f_{1}\right)$ were then demodulated to a low frequency $f_{1}$ using an SR-850 lock-in amplifier. Experimentally, we observed the demodulated signal both using an SR-785 (in frequency domain) and an oscilloscope (in time domain). Please note that the carrier frequency reduction technique mentioned in Ref. 12 was needed to reduce the magnitude of the carrier signal at the drive field frequency of $f_{0}$.

\section{RESULTS AND DISCUSSION}

Figure 2 shows the magnetostriction and effective linear piezomagnetic coefficient of the Metglas/PZT and Metglas/PMN-PT laminates for dc magnetic biases between -50 Oe and 50 Oe. As previously reported, ${ }^{19}$ and as can be seen in equations (2) and (3), it is actually not the magnetostriction coefficient that is important to the value of $\alpha_{M E}$, but rather that of effective linear piezomagnetic coefficient $d_{33}$, $m$. In this figure, the values of the magnetostriction and $d_{33, m}$ can be seen to be nearly the same for both types of laminates. However, some difference in $d_{33, m}$ can be seen between them at lower values of dc bias field $H_{\mathrm{dc}}$. The changes in
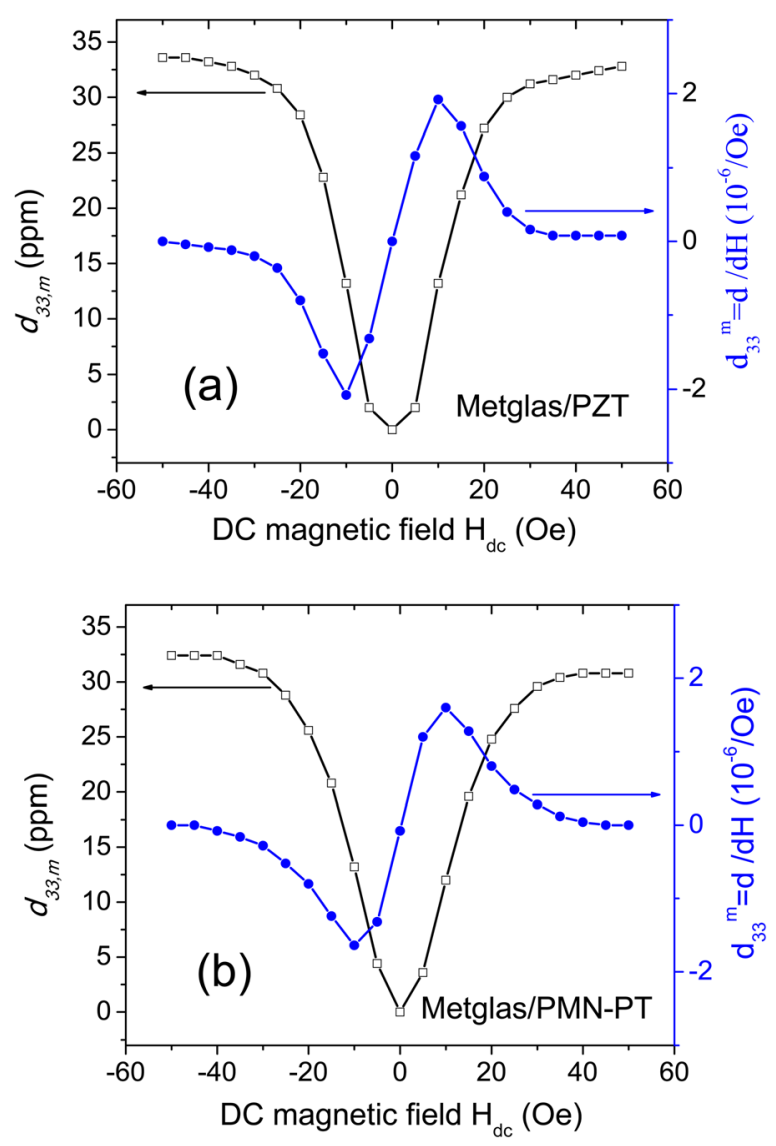

FIG. 2. (Color online) Magnetostriction and piezomagnetic properties of (a) Metglas/PZT and (b) Metglas/PMN-PT sensors. magnetostriction with $H_{\mathrm{dc}}$ were larger near $H_{\mathrm{dc}}=0$ for the Metglas/PMN-PT laminate than for the Metglas/PZT.

Figures 3(a) and 3(b) show the linear ME voltage coefficient $\alpha_{M E}$ as a function of $H_{\mathrm{dc}}$ for the Metglas/PZT and Metglas/PMN-PT laminates, respectively. The frequency of the incident field $H_{\text {inc }}$ was fixed at $f=1 \mathrm{kHz}$, but three different magnitudes of $H_{\text {inc }}(0.1 \mathrm{Oe}, 1 \mathrm{Oe}$, and $4 \mathrm{Oe})$ were applied. In these figures, a notable difference in the slope of $\alpha_{M E}$ near $H_{\mathrm{dc}}=0$ can be seen. For the laminate with PZT fibers, two different slope regimes were found for $H_{\mathrm{dc}} \leq| \pm 8 \mathrm{Oe}|$ (as identified by I and II in Fig. 3(a)). In particular, the slope near $H_{\mathrm{dc}}=0$ was much smaller. Whereas, for the laminate with PMN-PT single crystal fibers, no such regime of low slope was observed, rather only a single regime was found for $H_{\mathrm{dc}} \leq \mid \pm 8$ Oe $\mid$ that had a higher slope. These differences will result in important corresponding differences in the nonlinear ME coefficient $\alpha_{M E}^{\text {Nonlin }}$ between the two types of laminate, as will be shown below. In addition, Figs. 3(a) and 3(b) reveal some dependence of $\alpha_{M E}$ on $H_{\text {inc }}$. Prior investigations have not shown such a dependence on $H_{\text {inc }}$. Our findings indicate an inherent nonlinearity in $\alpha_{M E}$.

Further investigations of the nonlinearity of $\alpha_{M E}$ under different dc magnetic biases was then performed, as summarized in Figs. 3(c) and 3(d) for Metglas/PZT and Metglas/PMN-PT laminates, respectively. The magnitude of the incident field $H_{\text {inc }}$ was varied between 0.2 Oe and 4 Oe. Five different $H_{\mathrm{dc}}$ biases were chosen: $9.5 \mathrm{Oe}$ (maximum $\alpha_{M E}$ value), $4.8 \mathrm{Oe}, 3 \mathrm{Oe}, 2 \mathrm{Oe}$, and 1 Oe. If $\alpha_{M E}$ lacks nonlinearity, then at a given $H_{\mathrm{dc}}, \alpha_{M E}$ should be constant and independent of the incident field used. In other words, $\alpha_{M E} / \alpha_{M E}^{N o r m}$ should always be equal to one over the entire $H_{\text {inc }}$ range, where $\alpha_{M E}^{N o r m}$ is the value under $H_{\text {inc }}=0.2 \mathrm{Oe}$. However, Figs. 3(c) and 3(d) show that $\alpha_{M E} / \alpha_{M E}^{N o r m}$ does change with $H_{\text {inc }}$. The results demonstrate that $\alpha_{M E}$ for both Metglas/PZT and Metglas/PMN-PT laminates are nonlinear. Also, the nonlinearity in $\alpha_{M E}$ was mostly determined by the value of $H_{\mathrm{dc}}$. It is important to note that the nonlinearity was minimal under $H_{\mathrm{dc}}=9.5 \mathrm{Oe}$, where $\alpha_{M E}$ was maximum (compare with Figs. 3(a) and 3(b)) for both laminate types, respectively. These findings clearly establish that the nonlinearities are largest at lower bias levels, where the linear $\alpha_{M E}$ is small.

Next, we directly measured the nonlinear ME voltage coefficient $\alpha_{M E}^{\text {Nonlin }}$. These measurements were performed using the cross-modulation method discussed in Sec. II, where the incident field $H_{\text {inc }}$ had a frequency of $f_{1}=1 \mathrm{~Hz}$. Figure 4(a) shows $\alpha_{M E}^{\text {Nonlin }}$ for a Metglas/PZT laminate taken at $f_{0}=1$ $\mathrm{kHz}$. A small nonlinearity of $\alpha_{M E}^{\text {Nonlin }}=0.1 \mathrm{~V} / \mathrm{cm}^{-\mathrm{Oe}^{2}}$ was found for $H_{\mathrm{dc}}=9.5 \mathrm{Oe}$, near which bias field the linear coefficient $\alpha_{M E}$ was maximum. Please note that previous publications were based on this dc bias field to achieve an optimized operation point. ${ }^{5-10}$ However, a maximum nonlinearity of $\alpha_{M E}^{\text {Nonlin }}=1.2 \mathrm{~V} / \mathrm{cm}-\mathrm{Oe}^{2}$ was found under $H_{\mathrm{dc}}=4.8$ Oe. These results clearly demonstrate that an improvement of $\alpha_{M E}^{\text {Nonlin }}$ by a factor of $10 \times$ can be achieved by optimizing $H_{\mathrm{dc}}$. Figure 4(b) shows $\alpha_{M E}^{\text {Nonlin }}$ for a Metglas/PZT sensor taken near its EMR at $f_{0}=31 \mathrm{kHz}$. Under this drive condition, a maximum value of $\alpha_{M E}^{\text {Nonlin }}=25.5 \mathrm{~V} / \mathrm{cm}-\mathrm{Oe}^{2}$ was found: which is a $20 \times$ improvement compared to that at $f_{0}=1 \mathrm{kHz}$. Therefore, for the Metglas/PZT sensor, a total improvement of $255 \times$ was achieved by optimizing $H_{\mathrm{dc}}$ and $f_{0}$. 

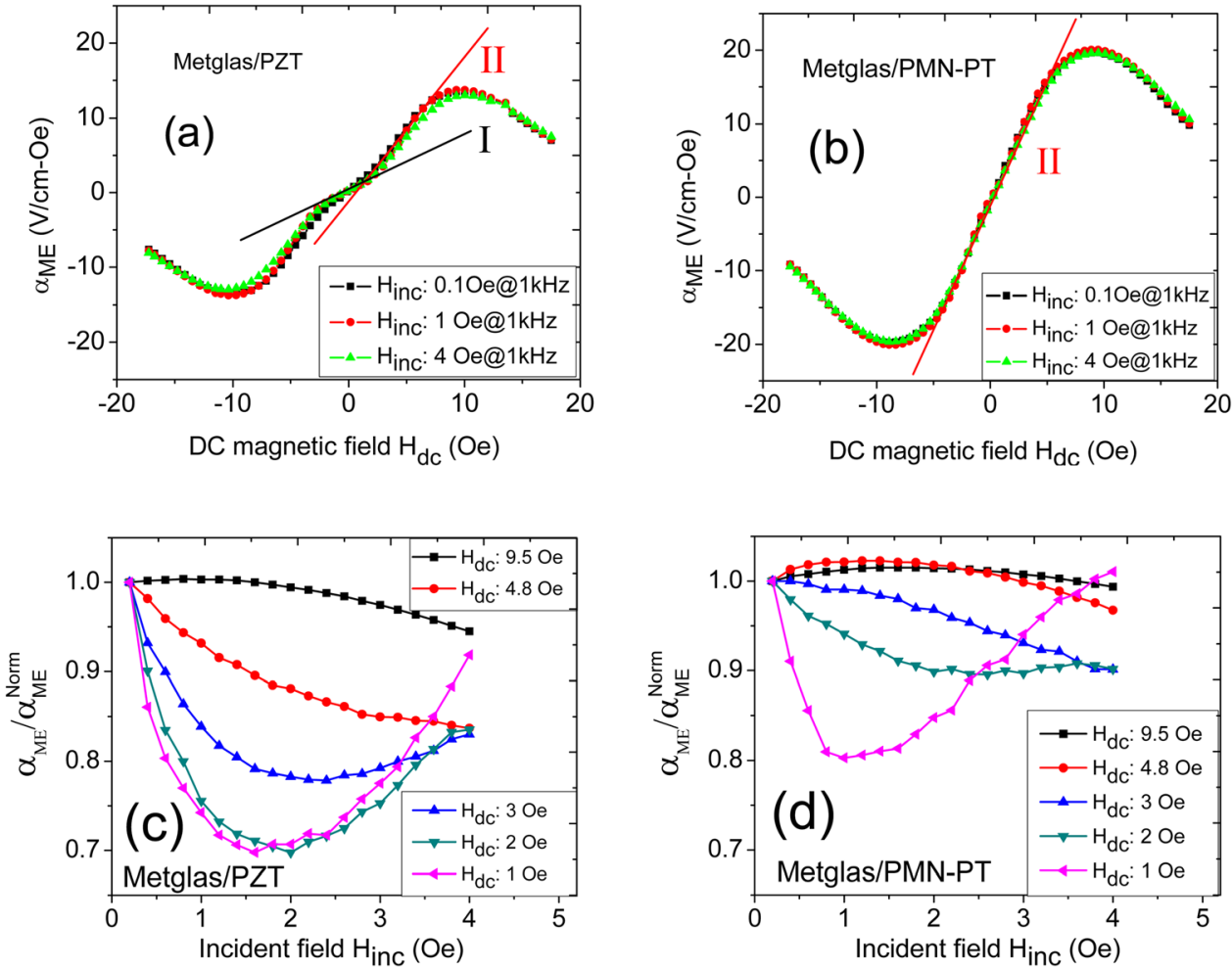

FIG. 3. (Color online) $\alpha_{M E}$ vs $H_{\mathrm{dc}}$ curves for (a) Metglas/PZT and (b) Metglas/PMN-PT sensors; $\alpha_{M E} / \alpha_{M E}^{\text {Norm }}$ vs $H_{\text {inc }}$ curves for (c) Metglas/PZT and (d) Metglas/PMN-PT sensors.
Figures 4(c) and 4(d) show similar measurements of $\alpha_{M E}^{\text {Nonlin }}$ for a Metglas/PMN-PT laminate at different frequencies. For $f_{0}=1 \mathrm{kHz}$ (see Fig. 4(c)), a minimum value of $\alpha_{M E}^{\text {Nonlin }}=0.1 \mathrm{~V} / \mathrm{cm}-\mathrm{Oe}^{2}$ was found near $H_{\mathrm{dc}}=9.5 \mathrm{Oe}$, whereas a much larger value of $\alpha_{M E}^{\text {Nonlin }}=2.2 \mathrm{~V} / \mathrm{cm}-\mathrm{Oe}^{2}$ was found under $H_{\mathrm{dc}}=2$ Oe. For $f_{0}=29.5 \mathrm{kHz}$ near the EMR (see Fig. 4(d)), this maximum value of $\alpha_{M E}^{\text {Nonlin }}$ was increased to 25.3 $\mathrm{V} / \mathrm{cm}^{-O e} .^{2}$ These results demonstrate a 253 times higher $\alpha_{M E}^{\text {Nonlin }}$ which was achieved by optimizing $H_{\mathrm{dc}}$ and $f_{0}$.
Furthermore, inspection of Figs. 4(c) and 4(d) will reveal that $\alpha_{M E}^{N o n l i n}$ is nearly maximum under $H_{\mathrm{dc}}=0$ Oe for Metglas/PMN-PT laminates: this result is much different than that for laminates with PZT fibers, where $\alpha_{M E}^{N o n l i n}$ was greatly reduced near $H_{\mathrm{dc}}=0$ Oe. These findings could have significant ramifications to the use of cross-modulation as detection methods for reduced noise, if the intrinsic sensor noise is not measured under an external dc magnetic bias. Not only can the low frequency noise floor be reduced for

\section{(a)}

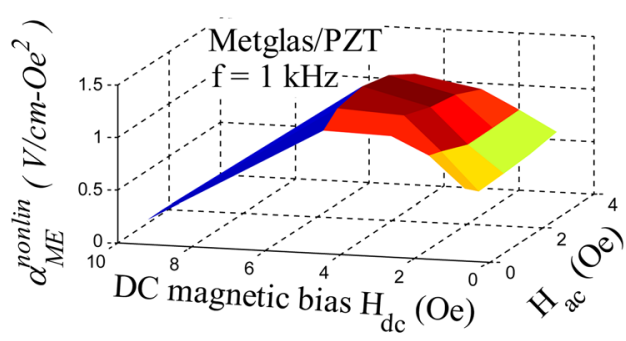

(c)

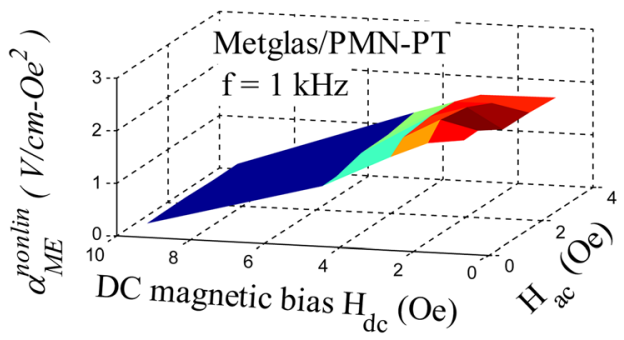

(b)

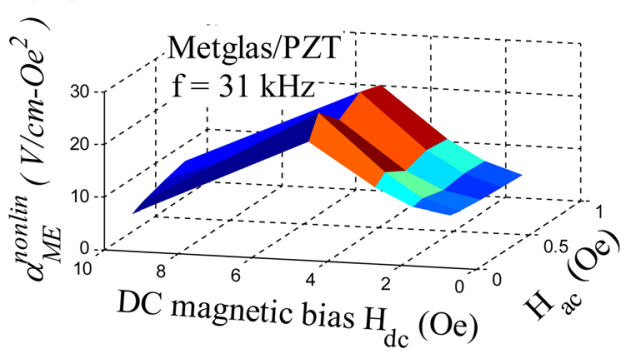

(d)

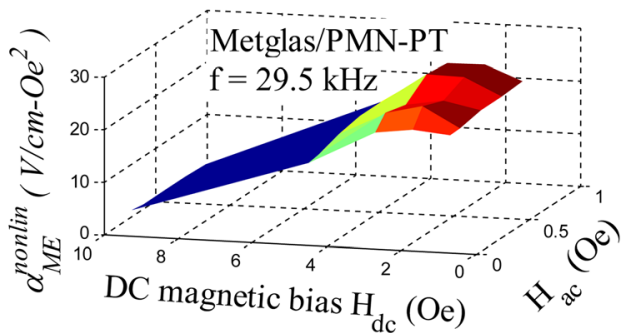

FIG. 4. (Color online) $\alpha_{M E}^{\text {Nonlin }}$ optimization of Metglas/PZT sensor at (a) $f_{0}=1$ $\mathrm{kHz}$ and (b) $f_{0}=31 \mathrm{kHz}(\mathrm{EMR}) . \alpha_{M E}^{\text {Nonlin }}$ optimization of Metglas/PMN-PT sensor at (c) $f_{0}=1 \mathrm{kHz}$ and (d) $f_{0}=29.5$ $\mathrm{kHz}(\mathrm{EMR})$, respectively. 
Metglas/PMN-PT by cross-modulation, but in addition its maximum performance can be designed to occur without a restriction of having a permanent $H_{\mathrm{dc}}$. The physical reason for this difference in $\alpha_{M E}^{\text {Nonlin }}$ between various piezoelectric materials is interesting and should be noted. Single crystals of PMN-PT oriented and poled along [001] are in a domainengineered state, ${ }^{21}$ which has a bridging monoclinic structure. $^{22,23}$ Hysteretic and dielectric losses are extremely small under both moderate bipolar and large unipolar drives in this domain engineered state, ${ }^{24}$ and thus minor hysteresis loops are negligible under these conditions. Application of the electric field does not result in domain wall motion, but rather the polarization is free to rotate in a plane. ${ }^{25}$ However, in conventional PZT piezoelectric ceramics, hysteretic losses are high and minor hysteresis loops known about $\mathrm{E}=0$ in the poled condition. ${ }^{24}$ Application of the electric field results in domain movement, where the field at which motion is induced can be notably influenced (i.e., pinned) by substituents and vacancies.

Figure 5 gives the test results for the Metglas/PMN-PT sensor using the cross-modulation scheme, where a $10 \mathrm{nT}$ incident field at a frequency of $f_{1}=1 \mathrm{~Hz}$ and a 0.5 Oe driving field at a frequency of $f_{0}=29.5 \mathrm{kHz}$ were applied. More information on the cross-modulation approach can be found in Ref. 12. Similar results were found for Metglas/PZT sensors, and thus are not shown: the only difference was that
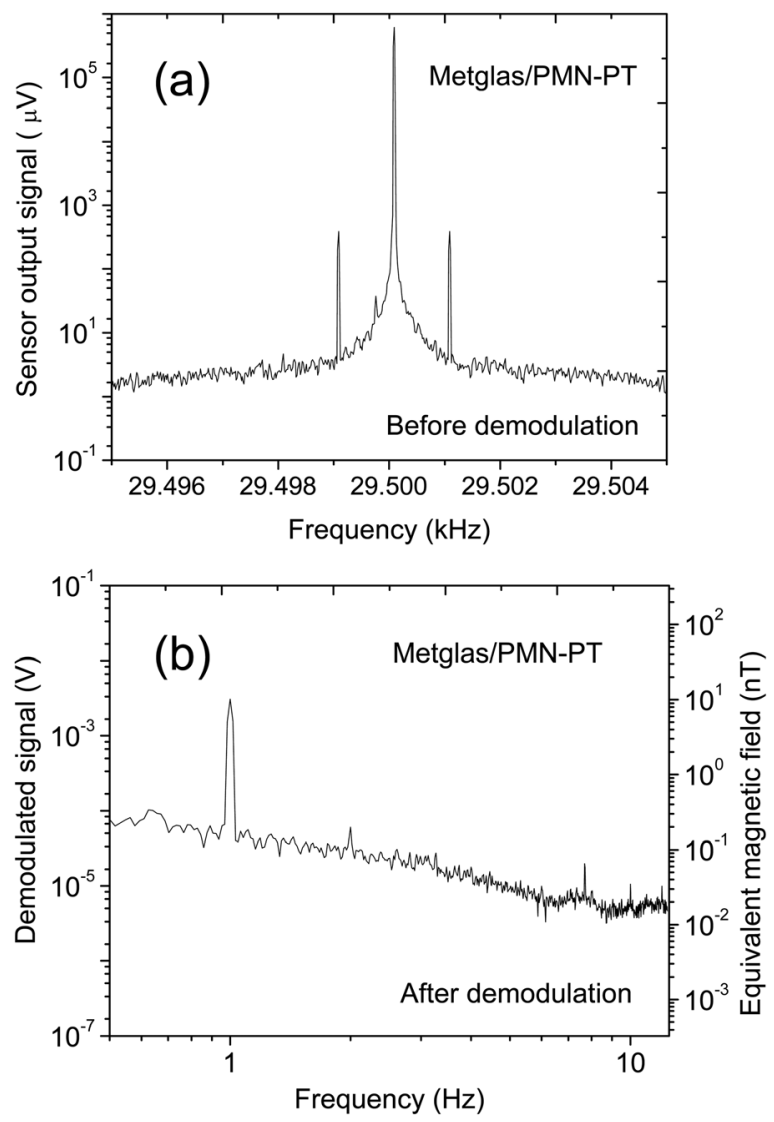

FIG. 5. Demonstrated test results of Metglas/PMN-PT sensors using crossmodulation scheme under $H_{\mathrm{dc}}=0$ Oe: (a) Sensor output signal before demodulation; (b) demodulated $1 \mathrm{~Hz}$ signal. the Metglas/PMN-PT sensors did not need a dc magnetic bias $H_{\mathrm{dc}}$ to achieve an optimized value of $\alpha_{M E}^{\text {Nonlin }}$. Figure 5(a) shows the sensor output signal before demodulation, which contains the carrier and cross-modulation signals at the frequencies of $29.5 \mathrm{kHz}\left(f_{0}\right), 29.499 \mathrm{kHz}$, and $29.501 \mathrm{kHz}$ $\left(f_{0} \pm f_{1}\right)$. Figure 5 (b) gives the demodulated $1 \mathrm{~Hz}$ magnetic field signal, as obtained by the SR-785 dynamic signal analyzer. This figure shows that the cross-modulation scheme has the ability of transferring low frequency magnetic signal $(1 \mathrm{~Hz})$ to higher frequencies $(29.499 \mathrm{kHz}$ and $29.501 \mathrm{kHz}$ ), where the noise floor is much lower than that at low frequency. ${ }^{26}$ Please note that the effectiveness of this frequency transfer is proportional to the nonlinear ME coefficient $\alpha_{M E}^{\text {Nonlin }}$. This is the reason why in this paper we tried to determine the conditions under which the nonlinear ME coefficient $\alpha_{M E}^{N o n l i n}$ was maximized.

Again, please bear in mind that the results in Fig. 5 were taken with no applied dc magnetic bias $H_{\mathrm{dc}}$. Since no dc bias is needed for the Metglas/PMN-PT sensor, it is a good candidate for sensor array and gradiometer applications, ${ }^{27,28}$ because there would be no dc magnetic bias interface among different sensors and therefore the sensor arrangement could be expected to be more flexible.

\section{SUMMARY}

We have performed an investigation of the nonlinear magnetoelectric properties of Metglas/PZT and Metglas/PMN-PT sensors. By optimizing $H_{\mathrm{dc}}$ and the drive frequency $f_{0}$, the nonlinear ME coefficient $\alpha_{M E}^{\text {Nonlin }}$ was increased by more than 250 times. We find that the value of $\alpha_{M E}^{\text {Nonlin }}$ is large under small $H_{\mathrm{dc}}$, where the linear coefficient $\alpha_{M E}$ is small; whereas, it is small under the bias where linear coefficient $\alpha_{M E}$ is maximum. We also find an important difference between laminates containing PMN-PT fibers as compared to those with PZT ones. This is that those with PZT fibers only have small values of $\alpha_{M E}^{\text {Nonlin }}$ near $H_{\mathrm{dc}}=0$ Oe, but those with PMN-PT have maximum values under this condition. The lack of dc bias requirements makes Metglas/PMN-PT sensors a good choice for applications that restrictions with regards to dc bias interference. Using the enhanced nonlinearity found in this study, together with a cross-modulation scheme recently reported, ${ }^{12}$ we have been able to reject low frequency noise in laminates, while not requiring dc magnetic biases.

\section{ACKNOWLEDGMENTS}

We acknowledge the support from the Defense Advanced Research Projects Agency (DARPA - BAA08 70) and assistance from Dr. Keith McLaughlin of SAIC.

${ }^{1}$ See http://www.darpa.mil/dso/solicitations/baa08-70.html for information about DARPA-BAA08-70, Heterostructural Uncooled Magnetic Sensors (HUMS).

${ }^{2}$ D. Robbes, C. Dolabdjian, S. Saez, Y. Monfort, G. Kaiser, and P. Ciereanu, IEEE Trans. Appl. Supercond. 11, 629 (2001).

${ }^{3}$ J. Vrba, SQUID Sensors: Fundamentals, Fabrication and Applications, edited by H. Weinstock (Kluwer Academic, Dordrecht, The Netherlands, 1996), p. 117.

${ }^{4}$ R. H. Koch, J. G. Deak, and G. Grinstein, Appl. Phys. Lett. 75, 3862 (1999). 
${ }^{5}$ L.D. Landau and E. Lifshitz, Electrodynamics of Continuous Media (Pergamon, Oxford, 1960), p. 119.

${ }^{6} \mathrm{~S}$. X. Dong, J.-F. Li, and D. Viehland, IEEE Trans. Ultrason. Ferroelectr. Freq. Control 50, 1253 (2003).

${ }^{7}$ S. X. Dong, J. Y. Zhai, J.-F. Li, and D. Viehland, Appl. Phys. Lett. 89, 252904 (2006).

${ }^{8}$ S. Trolier-McKinstry, N. Bassiri Gharb, and D. Damjanovic, Appl. Phys. Lett. 88, 202901 (2006)

${ }^{9}$ S. X. Dong, J. Y. Zhai, J.-F. Li, and D. Viehland, Appl. Phys. Lett. 89, 122903 (2006).

${ }^{10}$ M. Kläui, J. Phys. Condensed Matter 20, 313001 (2008).

${ }^{11}$ Ce-Wen Nan, Phys. Rev. B 50, 6082 (1994).

${ }^{12}$ X. Zhuang, M. Lam Chok Sing, C. Cordier, S. Saez, C. Dolabdjian, L. Shen, J. F. Li, M. Li, and D. Viehland, IEEE Sens. J. 11, 2266 (2011).

${ }^{13}$ J. Y. Zhai, Z. P. Xing, S. Dong, J. F. Li, and D. Viehland, Appl. Phys. Lett. 88, 062510 (2006).

${ }^{14}$ Z. P. Xing, J. Y. Zhai, S. X. Dong, J. F. Li, D. Viehland, and W. G. Odendaal, Meas. Sci. Technol. 19, 015206 (2008).

${ }^{15}$ X. Zhuang, M. Lam Chok Sing, C. Cordier, S. Saez, C. Dolabdjian, J. Das, J. Gao, J. F. Li and D. Viehland, IEEE Sens. J. 11, 2183 (2011).

${ }^{16}$ J. Y. Zhai, Z. P. Xing, S. Dong, J. F. Li, and D. Viehland, Appl. Phys. Lett. 93, 072906 (2008).
${ }^{17}$ Z. Xing, J. Zhai, J. F. Li, and D. Viehland, J. Appl. Phys. 106, 024512 (2009).

${ }^{18}$ C. C. Enz, E. A. Vittoz, and F. Krummenacher, IEEE J. Solid-State Circuits SC-22, 335 (1987).

${ }^{19}$ J. Das, J. Gao, Z. Xing, J.F. Li, and D. Viehland, Appl. Phys. Lett. 95, 092501 (2009).

${ }^{20}$ J. Zhai, Z. Xing, S. Dong, J. F. Li, and D. Viehland, J. Am. Ceram. Soc. 91, 351 (2008).

${ }^{21}$ S. Park and T. Shrout, J. Appl. Phys. 82, 1804 (1997).

${ }^{22}$ B. Noheda, Z. Zhong, D. Cox, G. Shirane, S. Park, and P. Rehrig, Phys. Rev. B 65, 224101 (2002).

${ }^{23}$ H. Cao, F. Bai, N. Wang, J. F. Li, D. Viehland, G. Xu, and G. Shirane, Phys. Rev. B 72, 064104 (2005).

${ }^{24}$ D. Viehland, J. Am. Ceram. Soc. 89, 775 (2006).

${ }^{25}$ D. Vanderbilt and M. Cohen, Phys. Rev. B 63, 094108 (2001).

${ }^{26}$ J. Gao, L. Shen, Y. Wang, D. Gray, J. F. Li, and D. Viehland, J. Appl. Phys. 109, 074507 (2011).

${ }^{27}$ Y. Shen, J. Gao, L. Shen, D. Gray, J. Li, P. Finkel, D. Viehland, X. Zhuang, S. Saez, and C. Dolabdjian, Sensors and Actuators A: Physical 171, 63 (2011).

${ }^{28}$ J. Gao, J. Zhai, Y. Shen, L. Shen, D. Gray, J. F. Li, P. Finkel, and D. Viehland, IEEE Trans. Ultrason. Ferroelectr. Freq. Control 58, 1541 (2011). 